\title{
Structure of continuum states and srength function in the complex scaling method
}

\author{
Myagmarjav Odsuren ${ }^{1, *}$, Gonchigdorj Khuukhenkhuu ${ }^{1}$, Sarsembayeva Aiganym ${ }^{2}$, Suren Davaa ${ }^{1}$, and Batsaikhan \\ Usukhbayar $^{1}$ \\ ${ }^{1}$ School of Engineering and Applied Sciences and Nuclear Research Center, National University of Mongolia, Ulaanbaatar 14200, \\ Mongolia \\ ${ }^{2}$ Department of Physics and Technology, Al-Farabi Kazakh National University, Almaty 050040, Kazakhstan
}

\begin{abstract}
We present our recent results of the mirror nuclei ${ }^{5} \mathrm{He}$ and ${ }^{5} \mathrm{Li}$ obtained by analyzing structure of continuum states in the complex scaled $\alpha+N$ two-body model. Decomposed scattering cross section and continuum level density of $\alpha+N$ system are discussed.
\end{abstract}

\section{Introduction}

We performed calculations using an $\alpha+\alpha+n$ three-cluster model together with the complex scaling method (CSM) [1, 2], which well reproduces the recentlyobserved photo-disintegration cross section [3]. The results indicated that the virtual state character of the $1 / 2^{+}$ state plays an important role in formation of the peak structure appearing in the cross section observed above the ${ }^{8} \mathrm{Be}+n$ threshold. However, the virtual state cannot be directly obtained as an isolated pole solution in the CSM, because the scaling angle in the CSM cannot be increased over the position of the virtual state pole on the negative imaginary axis of the complex momentum plane. A new approach for the CSM to describe the virtual state was proposed, and we discussed the pole position of the virtual state using the continuum level density, the scattering phase shift, and the scattering length calculated in the CSM [4]. The next problem is to calculate the contribution of the continuum state for partial waves with a resonance state.

For this purpose, we calculate the decomposed continuum level density and the decomposed scattering phase shifts of the low-lying states of ${ }^{5} \mathrm{Li}$ and ${ }^{5} \mathrm{He}$ mirror nuclei.

\section{Theoretical Framework}

\subsection{Complex scaling method}

In the CSM, the relative coordinate $\vec{r}$ is rotated as $\vec{r} \rightarrow \vec{r} e^{i \theta}$ in the complex coordinate plane. The complex-scaled Hamiltonian $H^{\theta}$ and wave function $\Psi_{J^{\pi}}^{v}(\theta)$ are defined as $U(\theta) H U^{-1}(\theta)$ and $U(\theta) \Psi_{J^{\pi}}^{v}$, respectively (see Ref.[2] for details). Therefore, the Schrödinger equation can be rewritten as

$$
H^{\theta} \Psi_{J^{\pi}}^{v}(\theta)=E_{v}^{\theta} \Psi_{J^{\pi}}^{v}(\theta),
$$

\footnotetext{
*e-mail: odsuren@seas.num.edu.mn
}

where $J^{\pi}$ is the spin and parity, $v$ is the state index, and $\theta$, being a real number, is the scaling angle.

Applying the $L^{2}$ basis function method, we expand the wave function as

$$
\Psi_{J^{\pi}}^{v}(\theta)=\sum_{n=1}^{N} c_{n}^{J^{\pi}, v}(\theta) \phi_{n}(r),
$$

where $\phi_{n}(r)$ and $c_{n}^{J^{\pi}, v}$ are the appropriate set of basis functions and expansion coefficients, respectively. The complex energy eigenvalues $E_{v}^{\theta}$ are obtained by solving the complex-eigenvalue problem given in Eq. (1). The complex energies of resonant states are obtained as $E_{r}=$ $E_{r}^{r e s}-i \Gamma_{r} / 2$, when $\tan ^{-1}\left(\Gamma_{r} / 2 E_{r}^{\text {res }}\right)<2 \theta$.

\subsection{Continuum level density}

The continuum level density $\rho(E)$ of the Hamiltonian $H$ is defined as a function of real energy $E$

$$
\rho(E)=\bigvee_{i} \delta\left(E-E_{i}\right)
$$

where $E_{i}$ are eigenvalues of $H$, and summation and integration are taken for discrete and continuous eigenvalues, respectively. This definition of the level density is also expressed using Green's function

$$
\rho(E)=-\frac{1}{\pi} \operatorname{Im}\left[\operatorname{Tr}\left\{\frac{1}{E+i 0-H}\right\}\right],
$$

where $+i 0$ indicates the limit $+i \epsilon \rightarrow+i 0$. When the Hamiltonian is described by a sum of an asymptotic term $H_{0}$ and the short-range interaction $V$, the continuum level density $\Delta(E)$ for an energy $E$ is expressed in terms of balance between the density $\rho(E)$ obtained from the Hamiltonian $H$ and the level density $\rho_{0}(E)$ of continuum states 
obtained from the asymptotic Hamiltonian $H_{0}$ as

$$
\Delta(E)=-\frac{1}{\pi} \operatorname{Im}\left[\operatorname{Tr}\left\{\frac{1}{E+i 0-H}-\frac{1}{E+i 0-H_{0}}\right\}\right] .
$$

The continuum level density is related to the scattering phase shift $\delta(E)$ and it can be expressed by the following form in the single channel case:

$$
\Delta(E)=\frac{1}{\pi} \frac{d \delta(E)}{d E} .
$$

Using this relation, we can obtain the phase shift as a function of the eigenvalues in the complex scaled Hamiltonian by integrating the continuum level density.

When we expand the wave functions in terms of the finite number $N$ of the basis states, the discretized eigenstates are obtained with number $N$ and the level density can be approximated by

$$
\begin{aligned}
\Delta(E) \approx & \Delta_{\theta}^{N}(E) \\
= & -\frac{1}{\pi} \operatorname{Im}\left[\sum_{b=1}^{N_{b}} \frac{1}{E+i 0-E_{b}}\right. \\
& +\sum_{r=1}^{N_{r}^{\theta}} \frac{1}{E-E_{r}^{r e s}+i \Gamma_{r} / 2} \\
& \quad \sum_{c=1}^{N_{c}^{\theta}} \frac{1}{E-\epsilon_{c}^{r}+i \epsilon_{c}^{i}} \\
& \left.\quad-\sum_{k=1}^{N} \frac{1}{E-\epsilon_{k}^{0 r}+i \epsilon_{k}^{0 i}}\right],
\end{aligned}
$$

where $N=N_{B}+N_{R}^{\theta}+N_{c}^{\theta}$ is the total number of $N_{B}$ (bound states), $N_{R}^{\theta}$ (resonance states) and $N_{c}^{\theta}$ (continuum states) solutions.

\section{Decomposition of scattering phase shifts and continuum level density}

The $\alpha-N$ system is described by using the Hamiltonians

$$
\begin{aligned}
H & =-\frac{5 \hbar^{2}}{8 M} \nabla^{2}+V_{\alpha-N}(r)+\hat{V}_{F}, \\
H_{0} & =-\frac{5 \hbar^{2}}{8 M} \nabla^{2},
\end{aligned}
$$

where for the $\alpha-N$ potential we use the so-called microscopic KKNN potential [5]. The pseudopotential $\hat{V}_{F}=$ $\lambda\left|\Phi_{P F}\right\rangle\left\langle\Phi_{P F}\right|$ is the projection operator to remove the Pauli forbidden states from the relative motions of $\alpha-N$.

We solve the complex scaled eigenvalue problems for the Hamiltonians of Eq. (8) with $\theta=15^{\circ}$ and $N=50$ as well. The calculated energy eigenvalues for the $s_{1 / 2}$, $p_{3 / 2}$ and $p_{1 / 2}$ waves of $\alpha-n\left({ }^{5} \mathrm{He}\right)$ system are presented in the lowest panels of Fig. 1. It can be seen from the lowest panels of Fig. 1, the $s_{1 / 2}$ wave has one Pauli forbidden state but no resonance. But one resonant pole of $\alpha-n$ system is obtained: $\left(E_{r}^{r e s}, \Gamma_{r}\right)=(0.74,0.59) \mathrm{MeV}$ for $p_{3 / 2}$ and $(2.10,5.82) \mathrm{MeV}$ for $p_{1 / 2}$, which are compared with the experimental data $\left(E_{r}^{r e s}, \Gamma_{r}\right)=(0.798,0.648) \mathrm{MeV}$ for $p_{3 / 2}$ and $(1.27,5.57) \mathrm{MeV}$ for $p_{1 / 2}$ [7]. Using these results and Eq. (7), we calculate not only the total continuum level density but also contributions of the resonance and continuum terms which are shown in the middle panels of Fig. 1. The $3 / 2^{-}$and $1 / 2^{-}$states have their respective peaks, although the peak of the $1 / 2^{-}$state is not sharp. The $1 / 2^{+}$ state has no peak and negative values due to its repulsive nature. The peaks in the continuum level density of $3 / 2^{-}$ and $1 / 2^{-}$states appear at the position with the width corresponding to the resonance energy and decay width.

Using the obtained continuum level density, we calculate the phase shift. In the top panels of Fig. 1, we show the decomposed phase shifts of the $3 / 2^{-}, 1 / 2^{-}$and $1 / 2^{+}$states together with experimental data. We can see a good agreement between theoretical and experimental results for each partial waves. The resonance phase shift of $3 / 2^{-}$increases rapidly due to the small decay width. Although $1 / 2^{-}$has a larger width, the phase shift of $1 / 2^{-}$shows a clear resonance behavior beyond $\pi / 2$. The continuum phase shifts of both states are very similar. This trend seems due to the same $p$ wave scattering and a small effect of the $\ell \cdot s$ force to the background states. The property of the scattering phase shifts is determined from a sum of resonance and continuum terms. Therefore, the observed resonances depend not only on resonant states as poles but also on the contribution from the non-resonant continuum states.

In a similar way as for the $\alpha-n$ case, we calculate the decomposed continuum level density and the decomposed scattering phase shifts of $\alpha-p\left({ }^{5} \mathrm{Li}\right)$ system, which includes the Coulomb interaction. Since the Coulomb interaction has a typically long-range character, the asymptotic Hamiltonian $H_{0}$ involves the Coulomb interaction. Using the obtained eigenvalues of $3 / 2^{-}, 1 / 2^{-}$and $1 / 2^{+}$states, we calculate the decomposed continuum level density and the decomposed scattering phase shifts in the same way as the calculations for ${ }^{5} \mathrm{He}$. The calculated results are shown in Fig. 2. The resonance solutions for $3 / 2^{-}$and $1 / 2^{-}$are obtained as $\left(E_{r}^{r e s}, \Gamma_{r}\right)=(0.78,0.55)$ and $(2.08,5.67)$ in $\mathrm{MeV}$, compared with the experimental data [8] $(1.53,1.42)$ and $(2.77,8.89)$ in $\mathrm{MeV}$, respectively.

These results indicate that the present method to calculate continuum level density is also powerful even for a long-range interaction such as the Coulomb potential.

\section{Summary}

Applying Green's function, we can precisely extract the contributions of resonance and continuum terms from the total continuum level density. This analysis clarifies the physical role of resonances and non-resonant continuum states in the observables. We have also shown the application of the CSM to the calculation of the decomposed continuum level density and the decomposed phase shifts. The role of resonance poles on the phase shifts and continuum level densities have been discussed. 


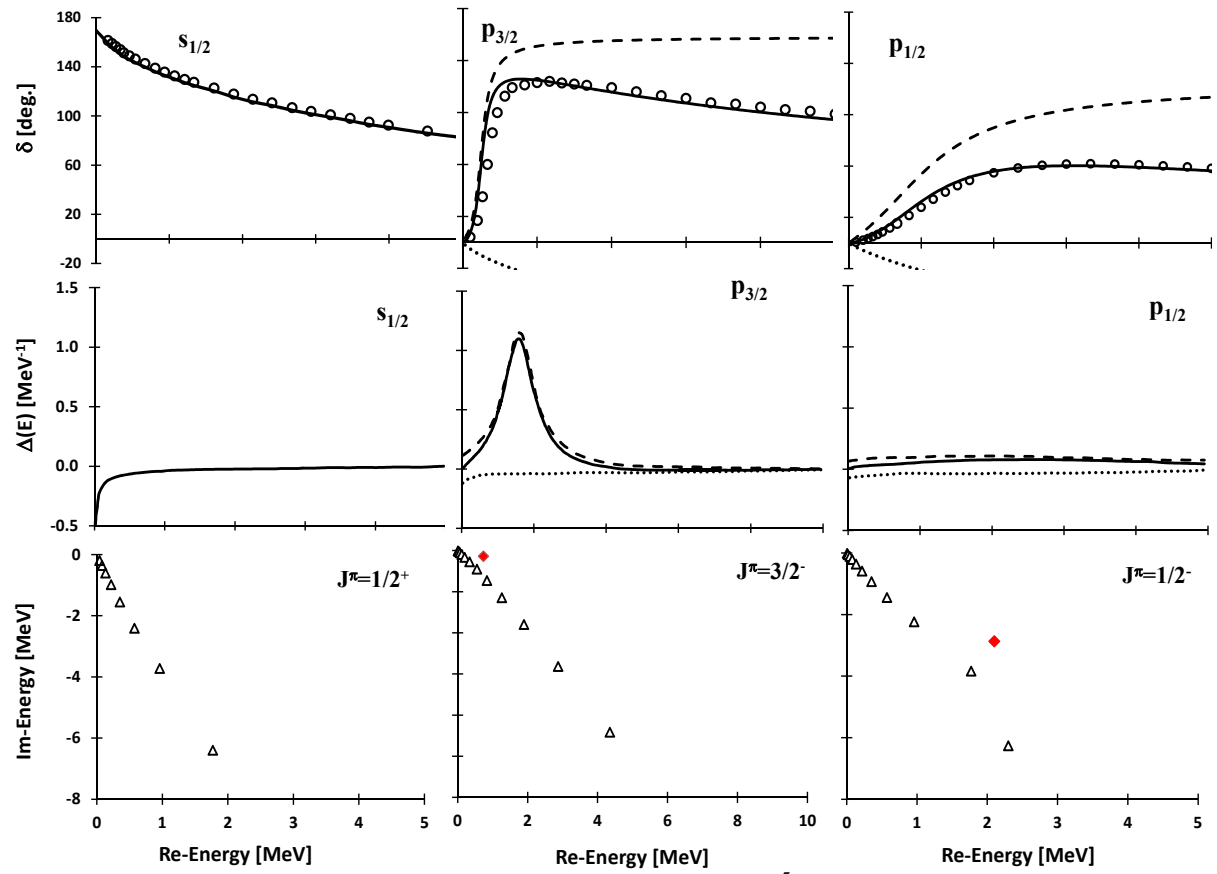

Figure 1. Upper panel: the decomposition of scattering phase shifts of $\alpha-n\left({ }^{5} \mathrm{He}\right)$ system for the $J^{\pi}=1 / 2^{+}, 3 / 2^{-}, 1 / 2^{-}$states and middle panel: the decomposition of continuum level densities. The dashed and dotted lines represent the contributions of resonance and continuum terms, respectively. The solid lines display total scattering phase shifts or total continuum level densities. Lower panel: the distributions of eigenvalues are displayed in the complex energy plane. The diamond displays the resonance pole. The experimental data [6] are shown with open circles.

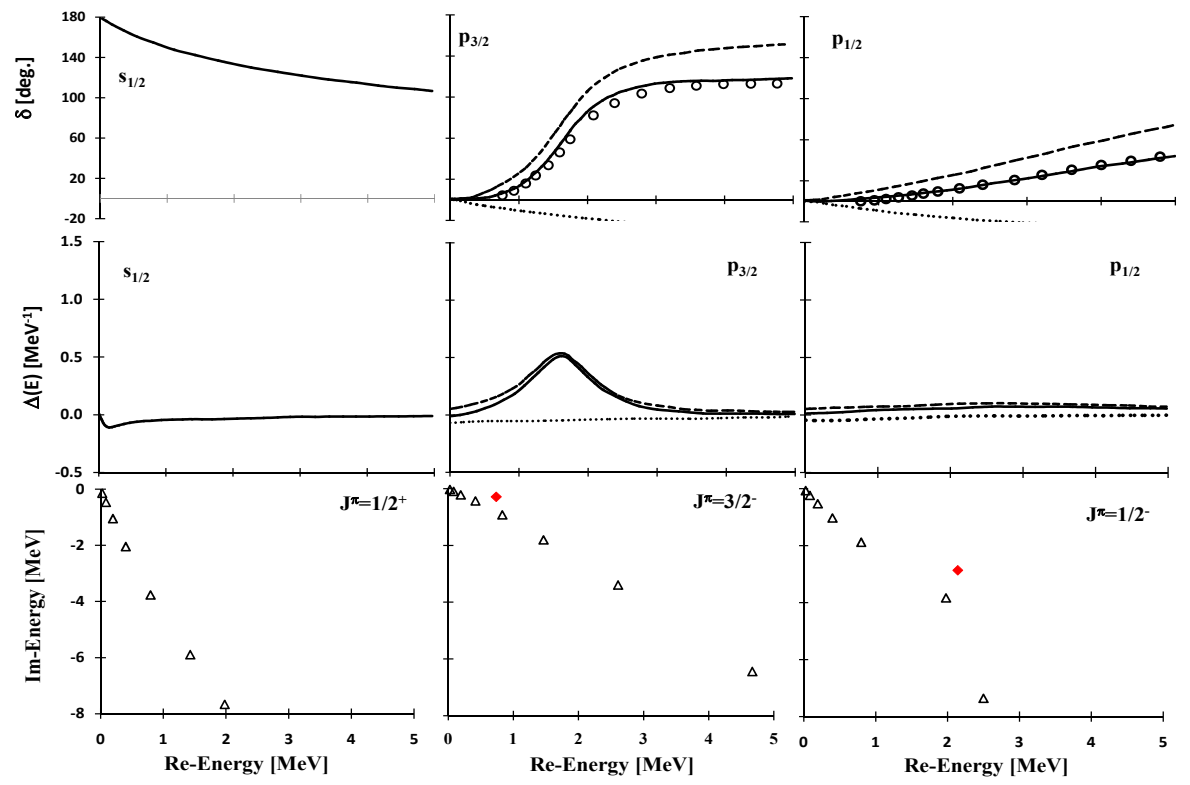

Figure 2. Same as Fig. 1 but for $\alpha-p\left({ }^{5} \mathrm{Li}\right)$ system. The experimental data [8] are shown with open circles.

\section{Acknowledgements}

This work was supported by National University of Mongolia's support for High impact research programm.

\section{References}

[1] K. Ho, Phys. Rep. 99, 1 (1983).
[2] S. Aoyama, T. Myo, K. Katō and K. Ikeda, Prog. Theor. Phys. 116, 1 (2006).

[3] M. Odsuren, Y. Kikuchi, T. Myo, M. Aikawa, and K. Katō, Phys. Rev. C 92, 014322 (2015).

[4] M. Odsuren, Y. Kikuchi, T. Myo, G. Khuukhenkhuu, H. Masui, and K. Katō, Phys. Rev. C 95, 064305 (2017). 
[5] H. Kanada, T. Kaneko, S. Nagata and M. Nomoto, Prog. Theor. Phys. 61, 1327 (1979).

[6] B. Hoop Jr and H. H. Barschall, Nucl. Phys. 83, 65 (1966); Th. Stammbach and R. L. Walter, Nucl. Phys. A180, 225 (1972). D. C. Dodder, G. M. Hale, N. Jarmie, J. H. Jett, P. W. Keaton, Jr., R. A. Nisley and K. Witte, Phys. Pev. C 15, 518 (1977); S. Ali, A. A.
Z. Ahmand and N. Ferdous, Rev. Mod. Phys. 57, 923 (1985).

[7] D. R. Tilley, C. M. Cheves, J. L. Godwin, G. M. Hale, H. M. Hofmann, J. H. Kelley, C. G. Sheu, H.R. Weller, Nucl. Phys. A708, 3 (2002).

[8] P. Schwandt, T. B. Clegg, and W. Haeberli, Nucl. Phys. A 163, 432 (1971). 